\title{
Management of peripheral nerve sheath tumors: 17 years of experience at Toronto Western Hospital
}

\author{
Daipayan Guha, MD, ${ }^{1}$ Benjamin Davidson, MD, ${ }^{1}$ Mustafa Nadi, MD, ${ }^{1}$ Naif M. Alotaibi, MD, ${ }^{1}$ \\ Michael G. Fehlings, MD, PhD, FRCSC, ${ }^{1,2}$ Fred Gentili, MD, MSc, ${ }^{1,2}$ Taufik A. Valiante, MD, PhD, ${ }^{1,2}$ \\ Charles H. Tator, MD, PhD, ${ }^{1,2}$ Michael Tymianski, MD, PhD, ${ }^{1,2}$ Abhijit Guha, MD, MSc, ${ }^{1,2}$ and \\ Gelareh Zadeh, MD, PhD ${ }^{1,2}$
}

\begin{abstract}
1'Department of Surgery, University of Toronto; and 2Division of Neurosurgery, Toronto Western Hospital, University Health
\end{abstract} Network, Toronto, Ontario, Canada

OBJECTIVE A surgical series of 201 benign and malignant peripheral nerve sheath tumors (PNSTs) was assessed to characterize the anatomical and clinical presentation of tumors and identify predictors of neurological outcome, recurrence, and extent of resection.

METHODS All surgically treated PNSTs from the Division of Neurosurgery at Toronto Western Hospital from 1993 to 2010 were reviewed retrospectively. Data were collected on patient demographics, clinical presentation, surgical technique, extent of resection, postoperative neurological outcomes, and recurrence.

RESULTS One hundred seventy-five patients with 201 tumors had adequate follow-up for analysis. There were 182 benign and 19 malignant PNSTs. Of the benign lesions, 133 were schwannomas, 21 of which were associated with a diagnosis of schwannomatosis. There were 49 neurofibromas, and 26 were associated with neurofibromatosis Type 1 (NF1). Patients presenting with schwannomas were significantly older than those with neurofibromas. Schwannomas were more readily resected than neurofibromas, with the extent of resection of the former influenced by tumor location. Patients with benign PNSTs typically presented with a painful mass and less frequently with motor deficits. The likelihood of worsened postoperative motor function was decreased in patients with fully resected tumors or preoperative deficits. Recurrence of schwannomas and neurofibromas were seen more frequently in patients diagnosed with NF3 and NF1, respectively. Subtotal resection was associated with the increased recurrence of all benign lesions.

CONCLUSIONS Outcomes following resection of benign PNSTs depend on tumor histopathology, tumor location, and genetic predisposition syndrome. Gross-total resection should be attempted for benign lesions where possible. The management of malignant PNSTs remains challenging, requiring a multimodal approach.

https://thejns.org/doi/abs/10.3171/2017.1.JNS162292

KEY WORDS peripheral nerve; schwannoma; neurofibroma; MPNST; tumor

$\mathrm{P}$ ERIPHERAL nerve tumors may be of neuroectodermal origin, derived from the neural sheath, or of nonneural sheath origin. Benign peripheral nerve sheath tumors (BPNSTs) include neurofibromas and schwannomas, which collectively constitute $10 \%-12 \%$ of benign soft-tissue neoplasms. ${ }^{19,28,29}$ Malignant PNSTs (MPNSTs) represent $5 \%-10 \%$ of all soft-tissue sarcomas and occur in $0.001 \%$ of the general population. ${ }^{12,30}$

Both BPNSTs and MPNSTs may occur sporadically, or in association with autosomal dominant neurofibromato- ses (NFs). Sixty percent of patients with neurofibromatosis Type 1 (NF1) - the most common NF-will develop solitary, diffuse, or plexiform benign neurofibromas, the latter two occurring almost exclusively in patients with NF1.11,26 NF1-associated neurofibromas have an estimated 10\%$15 \%$ risk of malignant transformation, with internal plexiform tumors at the highest risk of conversion..$^{32}$ MPNSTs occur in up to $10 \%$ of NF1 patients over their lifetimes; conversely, half of all MPNSTs are seen in NF1 patients. ${ }^{5}$ Peripheral schwannomas are typically sporadic, although

ABBREVIATIONS BPNST = benign peripheral nerve sheath tumor; GTR = gross-total resection; MPNST = malignant peripheral nerve sheath tumor; NF = neurofibromatosis; NF1, NF2, NF3 = NF Type 1, Type 2, Type 3; PNST = peripheral nerve sheath tumor; STR = subtotal resection.

SUBMITTED August 31, 2016. ACCEPTED January 26, 2017.

INCLUDE WHEN CITING Published online July 7, 2017; DOI: 10.3171/2017.1.JNS162292. 
up to $10 \%$ are associated with NF Type 2 (NF2). ${ }^{5}$ Rarely, patients may present with schwannomatosis, a largely sporadic disorder that is predisposed to nonvestibular nonintradermal schwannomas. ${ }^{21}$

Surgical excision remains the primary treatment modality for BPNSTs. Similar techniques are used for intraneural schwannomas, which arise from a single fascicle, and neurofibromas, which often have multiple thicker fascicles entering and exiting the tumor capsule. MPNSTs are treated best with wide surgical margins, followed by chemotherapy and local radiation; unfortunately, metastases are common, with poor long-term survival.

Here, we present a series of 201 benign and malignant PNSTs treated surgically over 17 years at Toronto Western Hospital. This represents one of the largest surgical series of PNSTs in the literature, ${ }^{1,15,23}$ which was collected over a relatively short period and predominantly treated by a single surgeon (A.G.) at a multidisciplinary institution with electrophysiologists, neurologists, and radiation and medical oncologists. The aim of the study is to identify predictors of neurological deficit, tumor recurrence, and extent of resection using multivariate analyses, as well as the impact of genetic predisposition syndromes on tumor location and frequency.

\section{Methods}

The charts of all patients with PNSTs who were treated surgically at the Division of Neurosurgery at Toronto Western Hospital between 1993 and 2010 were reviewed retrospectively. One hundred seventy-five patients with 201 primary tumors had adequate follow-up data for analysis. The mean follow-up time for all patients was 29.5 months. Institutional research ethics board approval was obtained from the University Health Network.

\section{Preoperative Workup}

All patients had their full medical histories evaluated (including family histories) and underwent general physical and neurological examinations, as well as screening for the signs of NF. Patients with the clinical findings of NF1 or NF2 were referred to a multidisciplinary NF clinic for screening neuroimaging, genetic testing, and counseling. MRI was performed on all cases. In patients with benign-appearing lesions on imaging, excisional biopsy was typically recommended for symptomatic lesions, lesions with interval growth during follow-up, or any lesions in NF1 patients that changed in size. In patients with suspected malignancy, which was often based on rapid clinical growth, irregular margins, and/or heterogeneous gadolinium enhancement on MR imaging, a preoperative needle or open biopsy was sometimes performed or intraoperative pathology was obtained to determine the need for wide excision.

\section{Surgical Technique}

Intraoperative nerve stimulation with electromyography recording was used in all procedures. Surgery for BPNSTs was performed according to established microsurgical principles. For schwannomas, fascicles adherent to the capsule were bluntly dissected off. One or some- times 2 fascicles were typically seen entering and leaving the tumor, and these were sectioned following electrophysiological confirmation of a lack of motor activity. The tumor was then removed en bloc. Larger tumors were enucleated via intracapsular dissection, followed by removal of the capsule if it was not densely adherent to the surrounding fascicles. Neurofibromas typically had 2 or more entering and exiting fascicles and required more careful dissection, but with the same principles as for schwannoma resection.

Surgical management of MPNSTs generally involved local excision with wide margins. Amputation was performed in 1 case, following intraoperative histological confirmation of the malignancy with negative preoperative workup for systemic metastases.

\section{Data Collection}

The impact of sex, age, NF diagnosis, tumor location, tumor size, and extent of resection on the recurrence of PNSTs was explored. Along with these parameters, the impact of preoperative motor or sensory deficits or neuropathic pain on the patients' respective postoperative outcomes was assessed. Tumor location was categorized as either the extremity (upper or lower) or paraspinal plexus (brachial, thoracic, or lumbosacral). Preoperative tumor size was volumetrically approximated as a cylinder based on the maximal in-plane dimensions. The extent of resection was classified as gross-total (GTR) resection or subtotal resection (STR) based on the intraoperative findings. For BPNSTs, GTR implied complete removal of the tumor capsule and parent fascicles; for MPNSTs, GTR was signified by negative surgical margins on frozen section and permanent pathology. Neuropathic pain was identified based on the patient reporting lancinating or dysesthetic pain in an identifiable nerve distribution. Postoperative motor/sensory/neuropathic pain scores were dichotomized as either stable/improved or worse relative to the preoperative status based on objective confrontational testing of motor function and patient self-reporting of sensory function and neuropathic pain.

All patients were followed up at 4-6 weeks for incision inspection and the first follow-up gadolinium-enhanced MRI at 3 months. The frequency of the subsequent MRI studies was variable, depending on the extent of resection and NF status. Tumor recurrence was defined as evidence of a new contrast-enhancing lesion on postoperative T1weighted MR images.

\section{Statistical Analysis}

Statistical comparison of the continuous variables was performed using the Student t-test; categorical variables were compared using the chi-square or Fisher's exact tests, as appropriate. Multiple logistic regression models were used to identify predictors of tumor recurrence, worsened postoperative motor/sensory/pain outcomes, as well as STR, with appropriate odds ratios and confidence intervals computed for each. Explanatory variables were incorporated into the model with forward stepwise progression; nonsignificant interaction terms were dropped to create the final models. The significance levels for all tests were 
set at $\mathrm{p}<0.05$. All statistical analyses were conducted using SPSS version 21 (IBM Corp.).

\section{Results}

\section{Patient and Tumor Characteristics}

In our cohort of 175 patients, the mean age at presentation was 45.2 years, and the patients with neurofibroma or MPNST were younger than the patients with schwannoma ( $\mathrm{p}<0.001$ ). In total, $54.9 \%$ of patients were male, with no difference in the sex distribution across tumor histopathologies (Table 1). Of the 201 tumors in our cohort, there were 49 neurofibromas, 133 schwannomas, and 19 MPNSTs. Twenty-six of the 49 neurofibromas were associated with NF1, and 21 schwannomas were associated with NF Type 3 (NF3) (Table 2). Sixteen of 19 MPNSTs in our series were in patients diagnosed with NF1. The tumor size at diagnosis was available for 57 schwannomas and 20 neurofibromas in our series, with average sizes of 3.86 $\times 4.89 \times 3.54 \mathrm{~cm}$ and $4.12 \times 6.00 \times 3.98 \mathrm{~cm}$, respectively (Table 1). The difference in the volumetric preoperative size between schwannomas and neurofibromas was not statistically significant $(\mathrm{p}=0.336)$.

The anatomical locations of all benign tumors in our cohort are shown in Table 2. Schwannomas were found most commonly in the brachial plexus, lower extremity, and lumbosacral plexus. Neurofibromas showed a similar localization, but with significantly fewer tumors located in the upper extremity. Twelve of the 49 neurofibromas in our series were plexiform. Four of the 19 MPNSTs were in the brachial plexus, and 6 were in the lower extremity.

Among BPNSTs, GTR was achieved less often for neurofibromas than schwannomas, in $44.9 \%$ versus $76.7 \%$ of cases (OR 0.25, 95\% CI 0.12-0.50; $\mathrm{p}<0.001)$. This dif-

TABLE 1. Demographic and clinical characteristics of 175 surgically treated patients with 182 BPNSTs and 19 MPNSTs

\begin{tabular}{cc}
\hline Characteristic & Value \\
\hline Age in yrs, mean \pm SD & $45.2 \pm 15.8$ \\
\hline Schwannoma & $48.4 \pm 15.6$ \\
\hline Neurofibroma & $36.6 \pm 12.8$ \\
\hline MPNST & $38.6 \pm 17.3$ \\
\hline Male/female sex ratio & $96: 79$ \\
\hline Schwannoma & $64: 51$ \\
\hline Neurofibroma & $22: 21$ \\
\hline MPNST & $10: 7$ \\
\hline NF status, $n(\%)$ & $37(21.1)$ \\
\hline NF1 & $3(1.7)$ \\
\hline NF2 & $8(4.6)$ \\
\hline NF3 & $158(90.3)$ \\
\hline Tumor histopathology, $n(\%)$ & $17(9.7)$ \\
\hline BPNST & \\
\hline MPNST & $66.86 \pm 115.46$ \\
\hline BPNST size in cm ${ }^{3}$, mean \pm SD & $98.39 \pm 150.32$ \\
\hline Schwannoma $(\mathrm{n}=57$ tumors $)$ & $29.5(1-302)$ \\
\hline Neurofibroma $(\mathrm{n}=20$ tumors) & \\
\hline Follow-up in mos, mean (range) & \\
\hline
\end{tabular}

ference persisted when plexiform neurofibromas were excluded; GTR was achieved for $51.4 \%$ of nonplexiform neurofibromas. Tumor location influenced the extent of resection specifically for schwannomas but not neurofibromas; schwannomas located in an extremity were more likely to be fully resected than plexal tumors (brachial/thoracic/ lumbosacral) (OR 3.36, 95\% CI 1.41-8.00; $\mathrm{p}=0.007)$, and schwannomas in the brachial plexus were more likely to be fully resected than those in the lumbosacral plexus (OR $4.76,95 \%$ CI $1.39-7.87 ; \mathrm{p}=0.01)$. NF status did not influence the extent of resection for any BPNSTs. GTR was significantly less likely to be achieved for MPNSTs than BPNSTs (OR 0.22, 95\% CI 0.078-0.60; $\mathrm{p}=0.002$ ); limbsparing surgery was performed in all but 1 patient with MPNST in our series.

\section{Motor, Sensory, and Pain Outcomes}

The symptomatic presentation of all 201 tumors in our series is summarized in Table 3 . In total, $12.8 \%$ of cases of schwannoma, $30.6 \%$ of cases of neurofibroma, and $36.8 \%$ of cases of MPNST presented with a preoperative motor deficits attributable to their PNST. Of these, all patients with schwannomas or neurofibromas remained stable or improved postoperatively, with permanent worsening seen in 2 of 7 MPNST patients (Fig. 1). New motor deficits were seen in 12 schwannoma patients (6 permanent) and 4 neurofibroma patients ( 3 permanent). Among the patients with a preoperative motor deficit, a diagnosis of MPNST was associated with an increased likelihood of worsened postoperative motor function relative to a diagnosis of BPNST (OR 29.55; 95\% CI 1.24-702.69; $p=0.028$ ); this association did not reach statistical significance for the entire cohort. In the univariate analyses, among BPNSTs, GTR (OR $4.01,95 \%$ CI 1.21-13.22; $\mathrm{p}=0.023$ ) and the presence of a preoperative motor deficit (OR 8.06, 95\% CI 4.65-17.59; $\mathrm{p}$ $=0.038$ ) were associated with an increased likelihood of stable/improved postoperative motor function. Only GTR was an independent predictor of stable/improved postoperative motor function in the multivariate analysis. Age, sex, tumor histopathology, NF status, location and tumor size were not significant predictors of postoperative motor outcome.

In total, $30.1 \%$ of patients with schwannomas, $44.9 \%$ of patients with neurofibromas, and $26.3 \%$ of patients with MPNSTs presented with paresthesia or numbness in a distribution relevant to their resected PNST. In total, 59.2\% had a positive Tinel's sign in the relevant nerve distribution. Postoperatively, the worsening of preexisting sensory disturbances was seen in 3 patients with schwannoma (1 permanent) and 2 patients with neurofibroma (both permanent). New sensory deficits were seen in 12 schwannoma patients ( 7 permanent), 1 neurofibroma patient $(0$ permanent), and 1 MPNST patient (permanent) (Fig. 1). In both the univariate and multivariate analyses, there were no significant predictors among our collected parameters that were associated with worsened postoperative sensory outcomes.

Pain was reported for $57.9 \%$ of schwannomas, $75.5 \%$ of neurofibromas, and $68.4 \%$ of MPNSTs. This pain was neuropathic in character and in the distribution of the affected nerve in $39.8 \%, 42.9 \%$, and $42.1 \%$ of schwannomas, 
TABLE 2. Anatomical locations of 182 BPNSTs

\begin{tabular}{|c|c|c|c|c|c|c|c|c|}
\hline \multirow[b]{2}{*}{ Location } & \multicolumn{4}{|c|}{ Schwannoma } & \multicolumn{3}{|c|}{ Neurofibroma } & \multirow[b]{2}{*}{ Total } \\
\hline & Sporadic & NF1 & NF2 & NF3 & Sporadic & NF1 & NF2 & \\
\hline \multicolumn{9}{|l|}{ Upper extremity } \\
\hline Axillary nerve & 2 & 0 & 0 & 0 & 0 & 0 & 0 & 2 \\
\hline \multicolumn{9}{|l|}{ Ulnar nerve } \\
\hline Elbow & 4 & 1 & 0 & 0 & 0 & 0 & 0 & 5 \\
\hline Wrist & 1 & 0 & 0 & 0 & 0 & 0 & 0 & 1 \\
\hline \multicolumn{9}{|l|}{ Median nerve } \\
\hline Arm & 5 & 0 & 0 & 0 & 1 & 0 & 0 & 6 \\
\hline Elbow & 4 & 0 & 0 & 0 & 1 & 0 & 0 & 5 \\
\hline Wrist & 0 & 0 & 0 & 1 & 0 & 0 & 0 & 1 \\
\hline \multicolumn{9}{|l|}{ Radial nerve } \\
\hline Arm & 1 & 0 & 0 & 0 & 0 & 0 & 0 & 1 \\
\hline Elbow & 2 & 0 & 0 & 0 & 0 & 0 & 0 & 2 \\
\hline PIN & 0 & 1 & 0 & 0 & 0 & 0 & 0 & 1 \\
\hline \multicolumn{9}{|l|}{ Brachial plexus } \\
\hline Supraclavicular & 21 & 0 & 2 & 3 & 4 & 7 & 0 & 37 \\
\hline Infraclavicular & 10 & 1 & 0 & 1 & 4 & 1 & 0 & 17 \\
\hline Thoracic paraspinal plexus & 1 & 0 & 0 & 6 & 1 & 1 & 0 & 9 \\
\hline \multicolumn{9}{|l|}{ Lower extremity } \\
\hline Femoral nerve & 2 & 0 & 0 & 0 & 2 & 5 & 0 & 9 \\
\hline Common sciatic nerve & 11 & 0 & 0 & 0 & 6 & 6 & 1 & 24 \\
\hline Tibial nerve & 8 & 0 & 0 & 3 & 1 & 0 & 0 & 12 \\
\hline Peroneal nerve & 19 & 0 & 0 & 1 & 1 & 1 & 0 & 22 \\
\hline Plantar nerve & 1 & 0 & 0 & 0 & 0 & 0 & 0 & 1 \\
\hline Sural nerve & 2 & 0 & 0 & 0 & 0 & 1 & 0 & 3 \\
\hline Lumbosacral plexus & 13 & 0 & 0 & 5 & 1 & 1 & 0 & 20 \\
\hline Occipital nerve & 0 & 0 & 0 & 1 & 0 & 2 & 0 & 3 \\
\hline Vagus nerve & 0 & 0 & 0 & 0 & 0 & 1 & 0 & 1 \\
\hline Total & 107 & 3 & 2 & 21 & 22 & 26 & 1 & 182 \\
\hline
\end{tabular}

PIN = posterior interosseous nerve.

neurofibromas, and MPNSTs, respectively. No patient with preoperative neuropathic pain reported worsening pain postoperatively. New postoperative neuropathic pain, in or adjacent to the distribution affected by the resected tumor, was reported by 2 patients with schwannoma (1 permanent), 2 patients with neurofibroma (1 permanent), and 2 patients with MPNST (1 permanent). None of our collected parameters, including tumor histopathology, were associated with worsened postoperative pain scores.

\section{Tumor Recurrence}

Among BPNSTs, the overall recurrence rates in our cohort were $5.3 \%$ for schwannomas and $8.2 \%$ for neurofibromas. Patients with recurrent schwannoma presented to neurosurgical attention on average 49.6 months after their index procedure, while patients with recurrent neurofibromas presented slightly earlier at 41.2 months. In the univariate analyses, a diagnosis of NF3 was associated with increased recurrence risk for schwannomas (OR $4.29,95 \%$ CI $1.05-20.80 ; p=0.048)$, with a recurrence rate of $14.3 \%$ in our cohort; a diagnosis of NF1 was associated with increased recurrence for neurofibromas (OR $1.18,95 \%$ CI 1.003-1.39; $\mathrm{p}=0.022$ ), with a recurrence rate of $15.4 \%$ in our cohort. In the multivariate analyses, STR

TABLE 3. Symptomatic presentation of 201 tumors

\begin{tabular}{lccccc}
\hline \multicolumn{1}{c}{ Tumor Type } & Weakness & Numbness/Paresthesia & Tinel's Sign & Pain & Mass Only \\
\hline Schwannoma & $17(12.8)$ & $40(30.1)$ & $86(64.7)$ & $77(57.9)$ & $30(22.6)$ \\
\hline Neurofibroma & $15(30.6)$ & $22(44.9)$ & $25(51.0)$ & $37(75.5)$ & $2(4.1)$ \\
\hline MPNST & $7(36.8)$ & $5(26.3)$ & $8(42.1)$ & $13(68.4)$ & $3(15.8)$ \\
\hline
\end{tabular}

All values reported as the number of tumors (\%). 

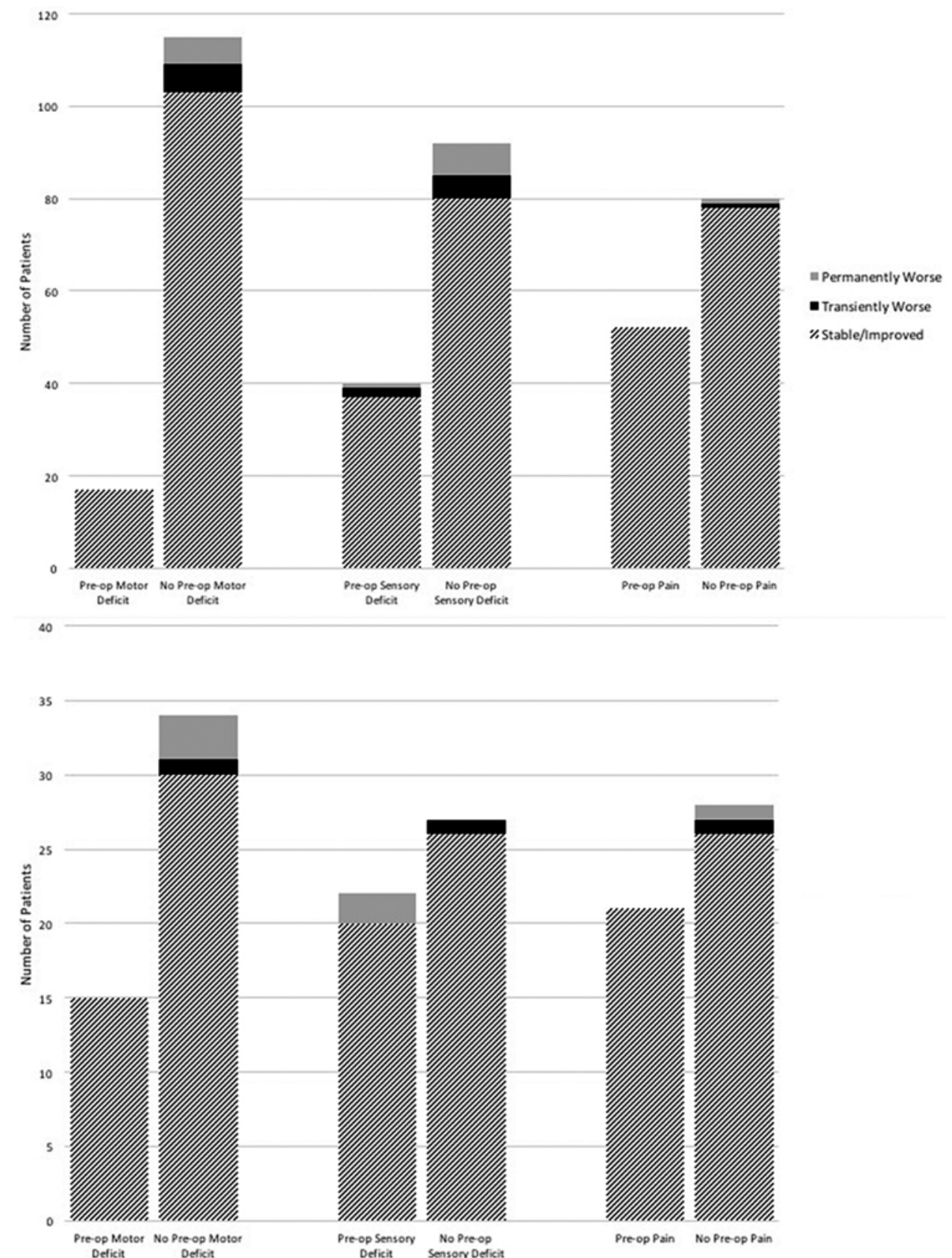

FIG. 1. Upper: Frequencies of the postoperative motor, sensory, and neuropathic pain outcomes for the 133 schwannomas in our series. Lower: Frequencies of the postoperative motor, sensory, and neuropathic pain outcomes for the 49 neurofibromas in our series. Outcomes are classified as stable/improved, transiently worse, or permanently worse relative to preoperative status.

was independently associated with increased recurrence for all BPNSTs (OR 13.16, 95\% CI 2.04-83.33; $\mathrm{p}=0.007$ ). Age, sex, tumor location, and tumor volume were not significant predictors of recurrence for BPNSTs.

The MPNSTs in our series showed a recurrence rate of $21 \%$, though a lack of neurosurgical follow-up may have diminished the true rate.

\section{Postoperative Complications}

New postoperative neurological deficits and neuropathic pain are summarized in Fig. 1. Among other compli- cations, wound infections occurred in 4 of 175 patients: 3 superficial infections requiring only oral antibiotics, and 1 deeper infection necessitating surgical washout. In 1 patient, a painful neuroma following resection of a schwannoma of the radial nerve at the elbow required resection at 1 year following the initial procedure. One patient with a plexiform neurofibroma of the brachial plexus experienced significant intraoperative bleeding, for which packing material was left in situ and removed during a second procedure. Dysphagia requiring temporary nasogastric tube feeding was seen in 1 patient following uni- 


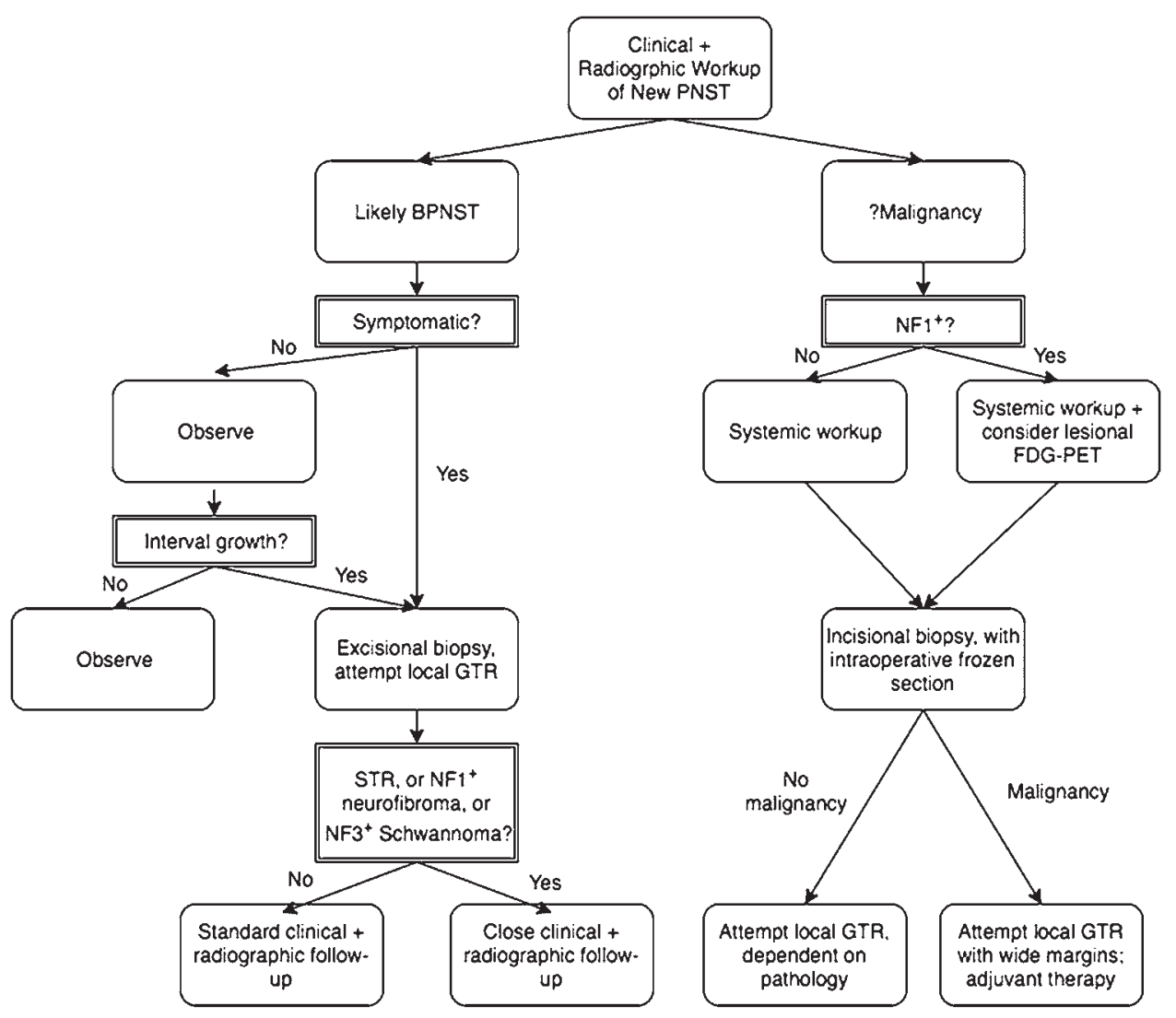

FIG. 2. A proposed decision algorithm for the management of PNSTs.

lateral vagus nerve sacrifice during resection of a brachial plexus neurofibroma. One patient experienced a CSF leak following resection of an infraclavicular brachial plexus neurofibroma, which resolved with lumbar drainage and over- sewing of the wound.

\section{Decision Algorithm}

Based on our, as well as other, large series, a surgical decision algorithm for PNSTs is proposed (Fig. 2). In our series, patients with a confirmed diagnosis of NF1, based on clinical and/or genetic criteria, were typically recommended for surgical excision of even asymptomatic benign-appearing PNSTs, with some variability based on lesion size.

\section{Discussion}

We present one of the largest single-institution series of symptomatic, surgically treated PNSTs. We aimed to identify predictors of tumor recurrence, extent of resection, and postoperative functional outcomes using multivariate analyses to identify independently associated parameters. To the best of our knowledge, this is only the second large series to explore predictors of outcome using multivariate statistical modeling.

Schwannomas and neurofibromas have a benign histology but may recur locally. The recurrence rates for BPNSTs range from $1.3 \%$ to $35.9 \%$ in the literature in $^{2,8,16,22,33}$ and up to $44 \%$ for pediatric plexiform neurofibromas. ${ }^{24}$ The recurrence rates for the schwannomas and neurofibro- mas in our series were $5.3 \%$ and $8.2 \%$, respectively. STR has been the most consistently identified predictor of recurrence and was independently and significantly associated with recurrence for both BPMSTs and MPNSTs in our series..$^{8,20,23}$

Other clinical, radiographic, and surgical parameters have been variably associated with recurrence or extent of resection. A diagnosis of NF1 has been associated with increased recurrence for neurofibromas in some series ${ }^{2,23}$ and STR of neurofibromas in others. ${ }^{6,15}$ Tumor histopathology has been shown to impact recurrence and extent of resection in some series. Artico et al. showed increased local recurrence in neurofibromas compared with schwannomas, and, in the largest reported series to date, Kim et al. demonstrated decreased GTR rates for neurofibromas, predominantly in their plexiform variants., ${ }^{2,15}$ Vetrano et al., however, found that patients with neurofibromas were at increased risk of postoperative neurological deficits but not recurrence; in their series, increased recurrence of BPNSTs was most associated with longer duration of symptoms prior to resection. ${ }^{35}$ Larger tumor size at diagnosis has also been associated with increased recurrence, though primarily in series of malignant PNSTs. ${ }^{27,39}$

In our series, tumor size was not significantly associated with recurrence. While the schwannomas on average were smaller at presentation than the neurofibromas in our cohort, this difference did not reach statistical significance and may be attributable to differences in tumor location. Proportionally fewer neurofibromas were located in the 
extremities, where they may be expected to be symptomatically or cosmetically noticeable at a smaller size in comparison with neurofibromas in a plexal location, which is consistent with other reports. ${ }^{19}$ We found that NF type was significantly associated with recurrence: NF1 for neurofibromas and schwannomatosis (i.e., NF3) for schwannomas. The increased recurrence risk for NF1-associated neurofibromas persisted when plexiform neurofibromas were excluded. Patients with hereditary predisposition syndromes should therefore be monitored more closely for recurrence. Whether a diagnosis of NF increases the likelihood of an asymptomatic BPNST that would require future resection is also uncertain. This question may be answered by longitudinal follow-up of the entire BPNST cohort. However, follow-up of asymptomatic patients in our cohort was largely relegated to primary care physicians, and hence only surgical outcomes are addressed here.

Interestingly, tumor location influenced the extent of resection only for schwannomas in our series, with schwannomas located in an extremity being more readily resected than plexal tumors likely due to anatomical accessibility. Schwannomas inherently grow extrinsic to their parent fascicles while neurofibromas are intertwined with multiple fascicles of origin and are therefore more difficult to fully resect irrespective of accessibility, which is a possible explanation for this finding. Another possibility is that the impact of tumor location is limited to schwannomas due to the disproportionately greater association of neurofibromas with NF. However, in our cohort 53\% of neurofibromas were associated with NF1, which is not significantly different from other series ${ }^{2,15,19,23}$; moreover, in multivariate modeling, tumor location influenced the extent of resection independently of NF status. Admittedly, our overall cohort of BPNSTs contains a higher number of patients with NF than other large series, largely due to the sizeable subset of schwannomas associated with NF3. However, our finding that NF3+ is associated with the increased recurrence of schwannomas has been echoed in other series..$^{13}$ Overall rates of GTR in our cohort were $44.9 \%$ for neurofibromas and $76.7 \%$ for schwannomas, which are lower than implied by Kim et al. in their large retrospective series. ${ }^{15}$ All patients in our series received intraoperative neurophysiological monitoring; Levi et al. reported an increase in the residual tumor in patients monitored intraoperatively, perhaps accounting for our lower rates. ${ }^{19}$ Unfortunately preoperative electrodiagnostic data were not available for all patients in our cohort.

Among the BPNSTs in our series, all patients with a preoperative motor deficit remained stable or improved postoperatively. New motor deficits were seen immediately postoperatively in $10.3 \%$ of schwannomas and $11.8 \%$ of neurofibromas, which were permanent in 5.2\% and 8.8\%, respectively. Preoperative sensory disturbances were permanently exacerbated in only 1 patient with schwannoma and 2 patients with neurofibroma. New sensory disturbances were seen immediately postoperatively in $12.9 \%$ of schwannomas and $3.7 \%$ of neurofibromas, which were permanent in $7.5 \%$ and $0 \%$, respectively. The rates of postoperative motor deficit in our cohort are largely in keeping with the lower end of the range reported in the literature. In the largest series to date, Kim et al. reported stable or improved motor function in $89 \%$ of schwannomas and $85 \%$ of solitary neurofibromas; ${ }^{15}$ Levi et al. reported postoperative motor deficits in $8 \%$ of schwannomas and $5.9 \%$ of neurofibromas..$^{19}$ In smaller series, permanent postoperative sensorimotor deficits have been reported in $11 \%-$ $36.7 \%$ of schwannoma patients. . $^{16,22,33}$

In our series, GTR and the presence of a preoperative motor deficit were predictive of stable or improved postoperative motor function. The latter is perhaps initially counterintuitive; however, patients with preexisting deficits attributable to a compressive lesion also have the potential to improve once their lesion is surgically decompressed. Ujigo et al. identified a preoperative positive Tinel's sign as predictive of postoperative sensorimotor deficit, but this was not observed in our series. ${ }^{33}$ Surgical technique may also influence postoperative neurological outcomes. Date et al. espoused the practice of intracapsular enucleation rather than extracapsular resection of schwannomas located in an extremity to minimize postoperative deficits. ${ }^{\text {? }}$ Levi et al. noted that patients with either schwannoma or neurofibroma who had undergone a needle or open biopsy procedure prior to definitive resection were at 2.7-fold higher risk of postoperative neurological deficits than those who underwent a resective procedure up front.$^{19}$ However, we were not able to identify in all records whether a biopsy had been performed at an outside institution.

MPNSTs represent a significant management challenge for peripheral nerve specialists. They occur mostly in the trunk and extremities but have been reported rarely at other sites..$^{25,36}$ Half are associated with NF1, with the remainder arising either de novo (40\%) or secondary to prior radiation (10\%). ${ }^{9,36}$ While MRI remains the initial workup of choice for all PNSTs, FDG-PET has shown promise for accurately differentiating benign from malignant NF1-associated lesions, potentially avoiding the morbidity associated with surgical biopsy. $3,10,37$

The primary treatment for MPNST remains wide local resection, though limb amputation in the absence of systemic metastases may be considered. Local recurrence rates may reach up to $65 \% \%^{4,39} ; 5$-year tumor-specific survival ranges from $16 \%$ to $52 \%$. $^{10,31,41}$ While NF1-associated MPNSTs have traditionally been associated with a poorer prognosis than sporadic lesions, ${ }^{18,39}$ a recent meta-analysis showed no significant differences in overall survival. ${ }^{17}$ Local recurrence for MPNST in our series was $21 \%$, well below the rates reported in the literature, though this likely reflects a loss of neurosurgical follow-up to oncological specialists. In multiple retrospective series, tumor size $(>$ $5 \mathrm{~cm}$ ), truncal location, incomplete resection, high grade, S100 $\beta$ negativity, and p53 mutations have been associated with worse prognosis. . $^{9,31,34,41}$ In our limited cohort of MPNSTs without immunohistopathological data available, STR was the only independent predictor of recurrence.

MPNSTs have traditionally been resistant to adjuvant chemo- or radiotherapy. Recent studies have demonstrated improved local control with adjuvant external-beam radiation but a negligible effect on overall survival.,14,34 The roles of proton-beam therapy and brachytherapy-the latter is applied widely with good effect on other soft-tissue sarcomas-have yet to be elucidated for MPNSTs. Conventional chemotherapy for MPNSTs has shown poor re- 
sults in trials on vincristine, doxorubicin, ifosfamide, and cyclophosphamide. ${ }^{40}$ Genetic and molecular alterations in MPNSTs may classify them into distinct strata, allowing the development of novel targeted therapeutics directed toward oncogenic pathways including mTOR, MEK, and VEGF. 4,38

\section{Conclusions}

We present here one of the largest surgical series of PNSTs to date. Functional outcomes are influenced by tumor histopathology, while recurrence rates depend on tumor histopathology, genetic predisposition syndrome, and, as demonstrated uniquely by our group, tumor location for certain subtypes. GTR should be attempted for benign lesions where feasible. MPNST remains a significant challenge, with a poor prognosis despite advances in multimodal therapies.

\section{References}

1. Anghileri M, Miceli R, Fiore M, Mariani L, Ferrari A, Mussi $\mathrm{C}$, et al: Malignant peripheral nerve sheath tumors: prognostic factors and survival in a series of patients treated at a single institution. Cancer 107:1065-1074, 2006

2. Artico M, Cervoni L, Wierzbicki V, D’Andrea V, Nucci F: Benign neural sheath tumours of major nerves: characteristics in 119 surgical cases. Acta Neurochir (Wien) 139:11081116, 1997

3. Benz MR, Czernin J, Dry SM, Tap WD, Allen-Auerbach MS, Elashoff D, et al: Quantitative F18-fluorodeoxyglucose positron emission tomography accurately characterizes peripheral nerve sheath tumors as malignant or benign. Cancer 116:451-458, 2010

4. Bradford D, Kim A: Current treatment options for malignant peripheral nerve sheath tumors. Curr Treat Options Oncol 16:328, 2015

5. Coffin CM, Davis JL, Borinstein SC: Syndrome-associated soft tissue tumours. Histopathology 64:68-87, 2014

6. Dafford K, Kim D, Reid N, Kline D: Pelvic plexus tumors. Neurosurg Focus 22(6):E10, 2007

7. Date R, Muramatsu K, Ihara K, Taguchi T: Advantages of intra-capsular micro-enucleation of schwannoma arising from extremities. Acta Neurochir (Wien) 154:173-178, 2012

8. Dozois EJ, Wall JCH, Spinner RJ, Jacofsky DJ, Yaszemski MJ, Sim FH, et al: Neurogenic tumors of the pelvis: clinicopathologic features and surgical outcomes using a multidisciplinary team. Ann Surg Oncol 16:1010-1016, 2009

9. Farid M, Demicco EG, Garcia R, Ahn L, Merola PR, Cioffi A, et al: Malignant peripheral nerve sheath tumors. Oncologist 19:193-201, 2014

10. Ferner RE, Golding JF, Smith M, Calonje E, Jan W, Sanjayanathan V, et al: $\left[{ }^{18} \mathrm{~F}\right] 2$-fluoro-2-deoxy-D-glucose positron emission tomography (FDG PET) as a diagnostic tool for neurofibromatosis 1 (NF1) associated malignant peripheral nerve sheath tumours (MPNSTs): a long-term clinical study. Ann Oncol 19:390-394, 2008

11. Ferner RE, Gutmann DH: Neurofibromatosis type 1 (NF1): diagnosis and management. Handb Clin Neurol 115:939955, 2013

12. Gupta G, Maniker A: Malignant peripheral nerve sheath tumors. Neurosurg Focus 22(6):E12, 2007

13. Halvorsen CM, Rønning P, Hald J, Johannesen TB, Kolstad F, Langmoen IA, et al: The long-term outcome after resection of intraspinal nerve sheath tumors: report of 131 consecutive cases. Neurosurgery 77:585-593, 2015

14. Kahn J, Gillespie A, Tsokos M, Ondos J, Dombi E, Camphau- sen K, et al: Radiation therapy in management of sporadic and neurofibromatosis type 1-associated malignant peripheral nerve sheath tumors. Front Oncol 4:324, 2014

15. Kim DH, Murovic JA, Tiel RL, Moes G, Kline DG: A series of 397 peripheral neural sheath tumors: 30-year experience at Louisiana State University Health Sciences Center. J Neurosurg 102:246-255, 2005

16. Kim SM, Seo SW, Lee JY, Sung KS: Surgical outcome of schwannomas arising from major peripheral nerves in the lower limb. Int Orthop 36:1721-1725, 2012

17. Kolberg M, Høland M, Agesen TH, Brekke HR, Liest $\varnothing 1 \mathrm{~K}$, Hall KS, et al: Survival meta-analyses for $>1800$ malignant peripheral nerve sheath tumor patients with and without neurofibromatosis type 1. Neuro Oncol 15:135-147, 2013

18. Kourea HP, Orlow I, Scheithauer BW, Cordon-Cardo C, Woodruff JM: Deletions of the INK4A gene occur in malignant peripheral nerve sheath tumors but not in neurofibromas. Am J Pathol 155:1855-1860, 1999

19. Levi AD, Ross AL, Cuartas E, Qadir R, Temple HT: The surgical management of symptomatic peripheral nerve sheath tumors. Neurosurgery 66:833-840, 2010

20. Li Q, Gao C, Juzi JT, Hao X: Analysis of 82 cases of retroperitoneal schwannoma. ANZ J Surg 77:237-240, 2007

21. MacCollin M, Chiocca EA, Evans DG, Friedman JM, Horvitz R, Jaramillo D, et al: Diagnostic criteria for schwannomatosis. Neurology 64:1838-1845, 2005

22. Mizushima H: Neurological deficits before and after surgical resection of schwannomas in the upper extremities. J Reconstr Microsurg 32:371-377, 2016

23. Montano N, D'Alessandris QG, D'Ercole M, Lauretti L, Pallini R, Di Bonaventura R, et al: Tumors of the peripheral nervous system: analysis of prognostic factors in a series with long-term follow-up and review of the literature. J Neurosurg 125:363-371, 2015

24. Needle MN, Cnaan A, Dattilo J, Chatten J, Phillips PC, Shochat $S$, et al: Prognostic signs in the surgical management of plexiform neurofibroma: the Children's Hospital of Philadelphia experience, 1974-1994. J Pediatr 131:678-682, 1997

25. Panigrahi S, Mishra SS, Das S, Dhir MK: Primary malignant peripheral nerve sheath tumor at unusual location. J Neurosci Rural Pract 4 (Suppl 1):S83-S86, 2013

26. Plotkin SR, Bredella MA, Cai W, Kassarjian A, Harris GJ, Esparza S, et al: Quantitative assessment of whole-body tumor burden in adult patients with neurofibromatosis. PLoS One 7:e35711, 2012

27. Rekhi B, Ingle A, Kumar R, DeSouza MA, Dikshit R, Jambhekar NA: Malignant peripheral nerve sheath tumors: clinicopathological profile of 63 cases diagnosed at a tertiary cancer referral center in Mumbai, India. Indian J Pathol Microbiol 53:611-618, 2010

28. Requena L, Sangüeza OP: Benign neoplasms with neural differentiation: a review. Am J Dermatopathol 17:75-96, 1995

29. Sangüeza OP, Requena L: Neoplasms with neural differentiation: a review. Part II: Malignant neoplasms. Am J Dermatopathol 20:89-102, 1998

30. Stark AM, Buhl R, Hugo HH, Mehdorn HM: Malignant peripheral nerve sheath tumours - report of 8 cases and review of the literature. Acta Neurochir (Wien) 143:357-364, 2001

31. Stucky CC, Johnson KN, Gray RJ, Pockaj BA, Ocal IT, Rose PS, et al: Malignant peripheral nerve sheath tumors (MPNST): the Mayo Clinic experience. Ann Surg Oncol 19:878-885, 2012

32. Tucker T, Wolkenstein P, Revuz J, Zeller J, Friedman JM: Association between benign and malignant peripheral nerve sheath tumors in NF1. Neurology 65:205-211, 2005

33. Ujigo S, Shimose S, Kubo T, Fujimori J, Ochi M: Therapeutic effect and risk factors for complications of excision in 76 patients with schwannoma. J Orthop Sci 19:150-155, 2014

34. Valentin T, Le Cesne A, Ray-Coquard I, Italiano A, Decanter 
G, Bompas E, et al: Management and prognosis of malignant peripheral nerve sheath tumors: The experience of the French Sarcoma Group (GSF-GETO). Eur J Cancer 56:77-84, 2016

35. Vetrano IG, Lucarella F, Dalolio M, Di Cristofori A, Nataloni IF, Tiberio F, et al: The importance of predicting factors in the surgical outcome of peripheral nerve sheath tumors. J Neurol Surg A Cent Eur Neurosurg 75:104-109, 2014

36. Wang J, Ou SW, Guo ZZ, Wang YJ, Xing DG: Microsurgical management of giant malignant peripheral nerve sheath tumor of the scalp: two case reports and a literature review. World J Surg Oncol 11:269, 2013

37. Warbey VS, Ferner RE, Dunn JT, Calonje E, O'Doherty MJ: $\left[{ }^{18} \mathrm{~F}\right] \mathrm{FDG}$ PET/CT in the diagnosis of malignant peripheral nerve sheath tumours in neurofibromatosis type-1. Eur J Nucl Med Mol Imaging 36:751-757, 2009

38. Watson MA, Perry A, Tihan T, Prayson RA, Guha A, Bridge $\mathrm{J}$, et al: Gene expression profiling reveals unique molecular subtypes of Neurofibromatosis Type I-associated and sporadic malignant peripheral nerve sheath tumors. Brain Pathol 14:297-303, 2004

39. Wong WW, Hirose T, Scheithauer BW, Schild SE, Gunderson LL: Malignant peripheral nerve sheath tumor: analysis of treatment outcome. Int J Radiat Oncol Biol Phys 42:351360, 1998

40. Zehou O, Fabre E, Zelek L, Sbidian E, Ortonne N, Banu E, et al: Chemotherapy for the treatment of malignant peripheral nerve sheath tumors in neurofibromatosis 1: a 10-year institutional review. Orphanet J Rare Dis 8:127, 2013

41. Zou C, Smith KD, Liu J, Lahat G, Myers S, Wang WL, et al:
Clinical, pathological, and molecular variables predictive of malignant peripheral nerve sheath tumor outcome. Ann Surg 249:1014-1022, 2009

\section{Disclosures}

The authors report no conflict of interest concerning the materials or methods used in this study or the findings specified in this paper.

\section{Author Contributions}

Conception and design: Zadeh, D Guha. Acquisition of data: D Guha, Davidson, Nadi, Fehlings, Gentili, Valiante, Tator, Tymianski. Analysis and interpretation of data: D Guha. Drafting the article: D Guha, Alotaibi. Critically revising the article: Zadeh, D Guha, Davidson, Nadi, Alotaibi. Reviewed submitted version of manuscript: Zadeh, Guha, Davidson, Nadi, Alotaibi, Fehlings, Gentili, Valiante, Tator, Tymianski. Approved the final version of the manuscript on behalf of all authors: Zadeh. Statistical analysis: D Guha. Administrative/technical/material support: Zadeh, Fehlings, Gentili, Valiante, Tator, Tymianski, A Guha. Study supervision: Zadeh.

\section{Correspondence}

Gelareh Zadeh, Division of Neurosurgery, Toronto Western Hospital, 4W-436, 399 Bathurst St., Toronto, ON M5T 2S8, Canada. email: gelareh.zadeh@uhn.ca. 